OPEN ACCESS

Edited by:

Zhongheng Zhang,

Sir Run Run Shaw Hospital, China

Reviewed by:

Yuli Huang Huang,

Southern Medical University, China

Shiyi Cao,

Tongji Medical College, China

${ }^{*}$ Correspondence:

Jun Lyu

Iyujun2020@jnu.edu.cn

Haiyan Yin

yinhaiyan1867@126.com orcid.org/0000-0002-9680-4219

†These authors have contributed equally to this work

Specialty section:

This article was submitted to Intensive Care Medicine and Anesthesiology,

a section of the journa

Frontiers in Medicine

Received: 09 March 2021 Accepted: 05 July 2021

Published: 26 July 2021

Citation:

Zhang L, Wang Z, Xu F, Ren Y,

Wang $H$, Han D, Lyu J and Yin $H$ (2021) The Role of Glucocorticoids in the Treatment of ARDS: A Multicenter

Retrospective Study Based on the

elCU Collaborative Research

Database. Front. Med. 8:678260. doi: 10.3389/fmed.2021.678260

\section{The Role of Glucocorticoids in the Treatment of ARDS: A Multicenter Retrospective Study Based on the elCU Collaborative Research Database}

\author{
Luming Zhang ${ }^{1,2+}$, Zichen Wang ${ }^{3 \dagger}$, Fengshuo X ${ }^{2,4}$, Yinlong Ren ${ }^{1}$, Hao Wang ${ }^{5}$, Didi Han ${ }^{2,4}$, \\ Jun Lyu ${ }^{2 *}$ and Haiyan Yin ${ }^{1 *}$
}

\footnotetext{
${ }^{1}$ Intensive Care Unit, The First Affiliated Hospital of Jinan University, Guangzhou, China, ${ }^{2}$ Department of Clinical Research, The First Affiliated Hospital of Jinan University, Guangzhou, China, ${ }^{3}$ Department of Public Health, University of California, Irvine, Irvine, CA, United States, ${ }^{4}$ School of Public Health, Xi'an Jiaotong University Health Science Center, Xi'an, China, ${ }^{5}$ Department of Statistics, lowa State University, Ames, IA, United States
}

Background: Acute respiratory distress syndrome (ARDS) is a common cause of respiratory failure in patients in intensive care unit (ICU). The therapeutic value of glucocorticoids (GCs) in the prognosis of ARDS remains controversial. The aim of this research is studying the impacts of GCs treatment on ARDS patients in ICU.

Methods: We retrospectively studied 2,167 ARDS patients whose data were collected from the public elCU Collaborative Research Database, among which 254 patients who received glucocorticoid (GCs) treatment were 1:1 matched by propensity matching analysis (PSM). The primary outcome was ICU mortality. Every oxygenation index (PaO2/FiO2) measurement before death or ICU discharge was recorded. A joint model (JM) which combined longitudinal sub-model (mixed-effect model) and time-to-event sub-model (Cox regression model) by trajectory functions of $\mathrm{PaO} 2 / \mathrm{FiO} 2$ was conducted to determine the effects of GCs treatment on both ICU mortality and $\mathrm{PaO} 2 / \mathrm{FiO} 2$ level and further PaO2/FiO2's effect on event status. The marginal structural cox model (MSCM) adjusted the overall $\mathrm{PaO} 2 / \mathrm{FiO} 2$ of patients to further validate the results.

Results: The result of the survival sub-model showed that GCs treatment was significantly associated with reduced ICU mortality in ARDS patients [HR (95\% Cl) $=0.642(0.453,0.912)]$, demonstrating that GCs treatment was a protective factor of ICU mortality. In the longitudinal sub-model, GCs treatment was not correlated to the $\mathrm{PaO} 2 / \mathrm{FiO} 2$. After adjusted by the JM, the HR of GCs treatment was 0.602 while GCs was still not significantly related to $\mathrm{PaO} 2 / \mathrm{FiO} 2$ level. The JM-induced association showed that higher $\mathrm{PaO} 2 / \mathrm{FiO} 2$ was a significant protective factor of mortality in ARDS patients and the HR was 0.991 which demonstrated that one level increase of PaO2/FiO2 level decreased $0.9 \%$ risk of ICU mortality. MSCM results also show that GCs can improve the prognosis of patients. 
Conclusion: Rational use of GCs can reduce the ICU mortality of ARDS patients in ICU. In addition to the use of GCs treatment, clinicians should also focus on the shifting trend of $\mathrm{PaO} 2 / \mathrm{FiO} 2$ level to provide better conditions for patients' survival.

Keywords: ARDS, glucocorticoids, ICU mortality, joint model, marginal structural cox model

\section{INTRODUCTION}

Acute respiratory distress syndrome (ARDS) has a high incidence and is one of the most common severe diseases in intensive care unit (ICU) $(1,2)$, which is a manifestation of lung parenchymal disease and represents various serious conditions, ranging from transient dyspnea to rapid respiratory failure (3). In the United States, about 200,000 patients are diagnosed with ARDS each year, and about 75,000 of them die. Globally, ARDS affects 3 million people every year, accounting for $10 \%$ of the ICU and $24 \%$ of the mechanically ventilated patients in the ICU (4). A vital early step in the inflammatory response to ARDS is recruiting macrophages (5), which then combine with the vascular endothelium to penetrate the vascular wall (pulmonary microvascular endothelial cells) and tissue (6), leading to extravascular accumulation of protein-filled edema fluid, which is a key pathophysiological mechanism of ARDS. Glucocorticoids (GCs) counteract lung injury by improving epithelial permeability, reducing edema, inhibiting local, systemic inflammation and reducing apoptosis (7). However, GCs use may also cause immunosuppression and drug resistance (8). Whether corticosteroids improve the prognosis of ARDS remains controversial (9), especially regarding their dosage and duration. At present, a large number of studies are further verifying the relationship between GCs and ARDS $(10,11)$. A randomized trial by Tomazini et al. showed that the use of intravenous dexamethasone can increase the survival days of patients with COVID-19 and moderate or severe ARDS (12). Another randomized study showed that dexamethasone reduced 28-day mortality in COVID-19 patients on mechanical ventilation (13). However, there are still many studies that have shown no beneficial effects of GCs use in patients with severe pulmonary infection or ARDS (14). For example, GCs use within 3 days of admission to the ICU has been shown to be associated with increased 90-day mortality in patients with COVID-19 (15). Applying the joint model (JM) to longitudinal and time to event data has become a valuable follow-up data analysis tool (16) that combines a linear mixed model with a Cox proportional hazard model based on trajectory function for longitudinal data and time-event data. Compared to the individual model, the JM provides a more effective way to predict how event status (survival) was impacted by treatment and estimate how longitudinal data, usually be as biomarkers, was impacted by treatment. In addition, the JM can induce the correlation between the biomarker and the event status. Compared to the individual model, JM can reduce the overall prediction bias and provides more accurate results. Therefore, this study intends to extract ARDS patients from the public eICU Collaborative Research Database (eICU), and use JM to investigate the effects of GCs on the level of $\mathrm{PaO} 2 / \mathrm{FiO} 2$ and survival of patients.

\section{MATERIALS AND METHODS}

\section{Data Source}

The data analyzed in this study were extracted from the eICU Collaborative Research Database, which is a public multicenter intensive care unit (ICU) database containing data on over 200,000 patients $(17,18)$. As a multi-center database, eICU contains electronic medical records among 208 hospitals from 2014-2015. All information about patient's identity is hidden, so the patient's informed consent is not required. The author had participated in a series of courses provided by NIH and obtained authorization to access the eICU database after passing the required assessment (certificate number 38601114). All data were extracted using SQL (Structured Query Language) before further analysis. The variables were longitudinal measurements of $\mathrm{PaO} 2 / \mathrm{FiO} 2$, age, sex, and race, $\mathrm{BMI}$, first care unit, ventilator use, vasopressor use, continuous renal replacement therapy (CRRT), SOFA score, and APACHE IV score.

\section{Study Population}

This study initially included 2,167 patients diagnosed with ARDS. After excluded patients without an APACHE IV score, patients with ICU stay shorter than $24 \mathrm{~h}$ and patients with abnormal $\mathrm{PaO} 2$ or FiO2 values, 1,557 patients remained, among which 254 received GCs. After 1:1 propensity score matching (PSM) for GCs treatment status based on demographic data including age, sex, and race, 508 patients were eventually selected for the study (Figure 1). The primary study outcome was ICU mortality, the secondary outcome was the effect of GCs on $\mathrm{PaO} 2 / \mathrm{FiO} 2$.

\section{STATISTICAL ANALYSIS}

\section{Longitudinal Data Analysis in JM}

The longitudinal data were analyzed by linear mixed effect model (Formula.1) in which $\mathrm{Y}_{\mathrm{ij}}$ represents the longitudinal outcome value of ith patient at the jth time of observation. $\mathrm{X}_{\mathrm{ij}}$ represents the trajectory of $\mathrm{X}$ of ith patient at the jth time of observation and $R_{i j}$ is the random effect of ith patient at the jth time and K's represented other functions of co-variants of $\mathrm{Y}$ while $\xi$ represents the coefficient (16). Every $\mathrm{PaO} 2 / \mathrm{FiO} 2$ measurement was observed for each patient before death or ICU discharge. Linear mixed-effects models were modeled by the trajectory function and random effects to analyze the longitudinal data. The dependent variable was $\mathrm{PaO} 2 / \mathrm{FiO} 2$ levels, and the independent variables were $\mathrm{PaO} 2 / \mathrm{FiO} 2$ at admission, GCs treatment and its two-way interaction with observational time to accurately reflect the relationship between GCs treatment and trend of $\mathrm{PaO} 2 / \mathrm{FiO} 2$ level for each patient (16). Adjustments of random effects were set as the observational time in order to minimize the random noises.

$$
Y_{\mathrm{ij}}=X_{\mathrm{ij}}+\xi K_{\mathrm{i}}+R_{\mathrm{ij}}
$$




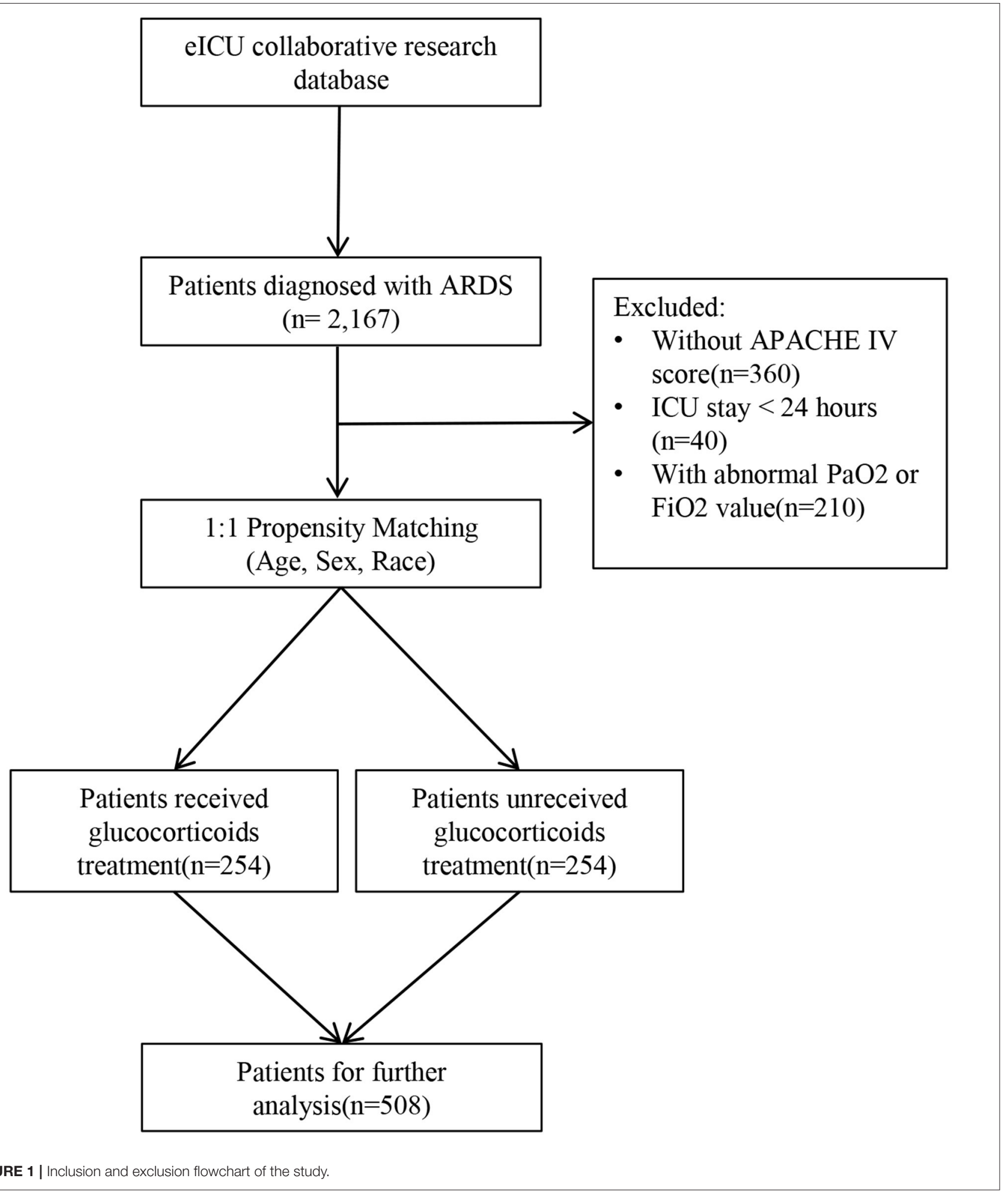

\section{Trajectory Function in JM}

Sub-models in JM are combined by trajectory function (Formula.2). The $\mathrm{X}_{\mathrm{ij}}$ represents the trajectory of longitudinal biomarker and the $\mathrm{Z}_{\mathrm{i}}$ represents the treatment (GCs) for ith patients. The trajectory function represents the linear relationship between observation times for patients $t_{i j}+$ treatment and the trajectory of biomarker $(\mathrm{PaO} 2 / \mathrm{FiO} 2) . \mathrm{C}_{0 \mathrm{i}}$ and $\mathrm{C}_{\mathrm{li}}$ are considered to be random.

$$
X_{\mathrm{ij}}=C_{0 \mathrm{i}+} C_{\mathrm{li}} \times t_{\mathrm{ij}}+\gamma Z_{\mathrm{i}}
$$




\section{A}

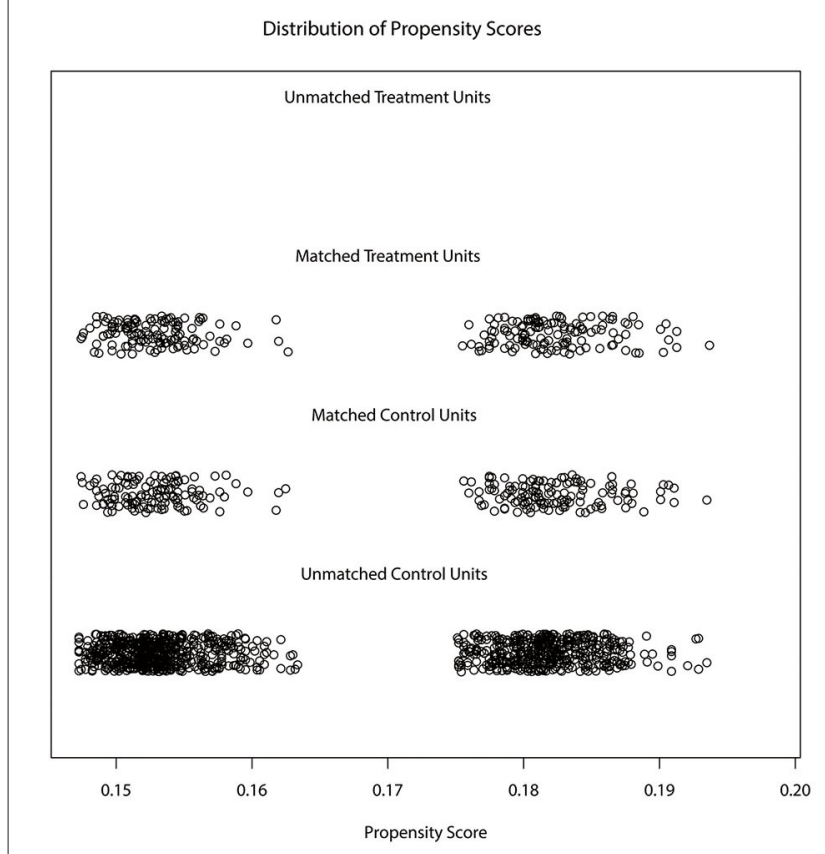

B
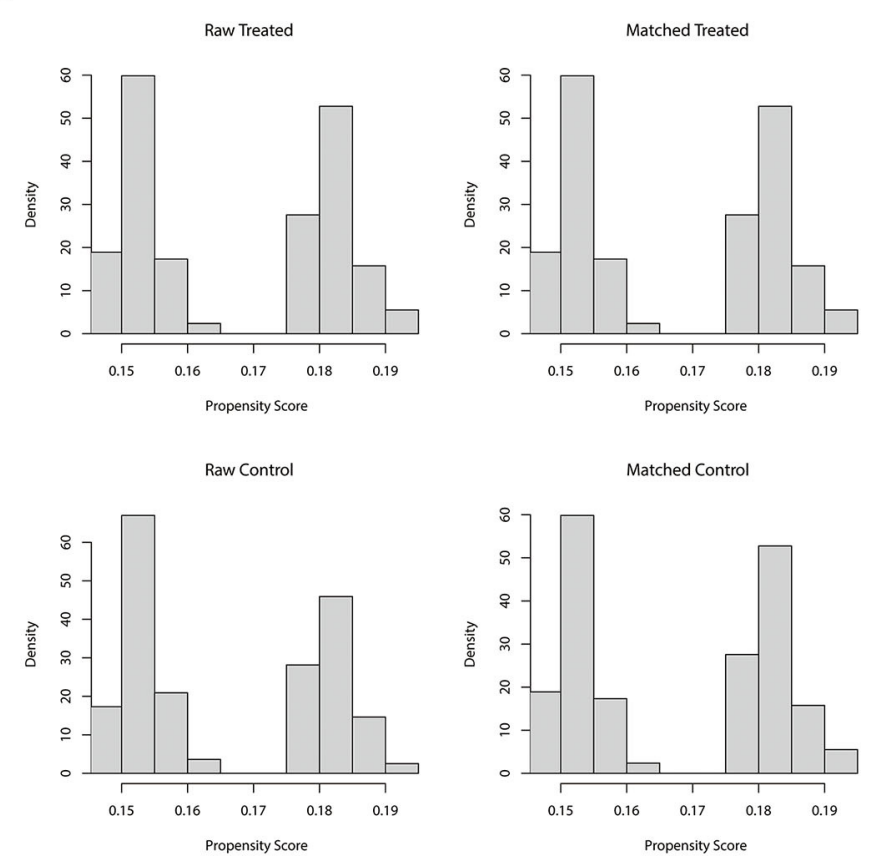

FIGURE 2 | Distribution of 1:1 propensity matching score in cluster (A) and histogram (B) match was based on demographic information included age, sex, and race.

\section{Time-to-Event Data Analyses in JM}

The time-to-event data were analyzed by the Cox proportionalhazards model (Formula.3). The $\mathrm{H}(\mathrm{t})$ represents the hazard function of ith patient at $\mathrm{t}, \mathrm{H}_{0}(\mathrm{t})$ represents the baseline hazard at t. $\beta$ and $\alpha$ represents the coefficient of trajectory for ith patient and coefficient of treatment indicator at for ith patients and K's represents other co-variables and $\tau^{\prime} s$ represents the coefficents.Compared to traditional Cox regression (Formula.4), the JM model not only reflects the relationship between treatment and both event and longitudinal biomarker, but also induces coefficient between biomarker and events. To determine the association between GCs treatment and ICU mortality. Cox proportional risk regression analysis was adjusted for imbalance variables between the two groups.

$$
\begin{gathered}
H(t)=H_{0}(t) \exp \left(\beta X_{\mathrm{ij}}+\alpha Z_{\mathrm{i}+} \tau K_{\mathrm{i}}\right) \\
H(t)=H_{0}(t) \exp \left(\alpha Z_{\mathrm{i}+} \tau K_{\mathrm{i}}\right)
\end{gathered}
$$

\section{Marginal Structural Cox Model}

Whether or not patients with ARDS received GCs during ICU hospitalization was considered a time-dependent variable in MSCM. Potential baseline confounding factors such as age, sex, race, $\mathrm{BMI}$, ICU unit type, ventilator use, vasopressor use, CRRT, SOFA, and Apache IV were obtained within $24 \mathrm{~h}$ of admission to the ICU. $\mathrm{PaO} 2 / \mathrm{FiO} 2$ throughout ICU hospitalization was included in the model as a time-varying confounding factor. The parameters of the MSCM can be estimated using inverse probability of treatment weighing (IPTW). The gradient boosted model (GBM) is a machine learning algorithm that involves an iterative process using multiple regression trees to provide more accurate estimates of response variables (19). IPTW based on this algorithm has been proved to be effective and robust in the study of two treatments. Therefore, in this paper, we use this algorithm to weight each patient and generate two virtual populations. The standardized mean differences (SMDs) $<0.2$ between the two groups was considered to have no significant difference in baseline characteristics (20). Then Cox regression was conducted again to further prove the validity of the results. Continuous variables were described as median and interquartile values based on their normality and $P$-values were calculated by Kruskal-Wallis test. Categorical variables were described as number and percentage values, with $P$-values calculated using chi-square tests. All statistical analyses were conducted using $\mathrm{R}$ software, the JM was constructed using the "JM" package, and the IPTW was constructed using the "twang" package. A two-side $p$-value $<0.05$ was considered statistically significant.

\section{RESULTS}

\section{Patient Characteristics}

Among the 508 ARDS patients after PSM, 254 patients received GCs treatment (Figure 2). Table 1 lists the baseline characteristics of the between GCs groups. The result showed that after the match for demographic information, patients between GCs groups were different from first admitted $\mathrm{PaO} 2 / \mathrm{FiO} 2$ and ventilator use. 
TABLE 1 | Baseline characteristics between GCs unreceived/received group.

\begin{tabular}{|c|c|c|c|}
\hline & GCs unreceived & GCs received & $p$ \\
\hline N & 254 & 254 & \\
\hline Age (year) & $60.00(49.00,69.00)$ & $59.00(49.00,69.00)$ & 0.655 \\
\hline Sex (\%) & & & 1.000 \\
\hline Male & $125(49.2)$ & 125(49.2) & \\
\hline Female & $129(50.8)$ & $129(50.8)$ & \\
\hline Race (\%) & & & 0.080 \\
\hline White & $189(74.4)$ & $178(70.1)$ & \\
\hline Black & $21(8.3)$ & $37(14.6)$ & \\
\hline Others & 44 (17.3) & $39(15.4)$ & \\
\hline BMl & $28.76(24.39,36.81)$ & $29.10(24.52,35.58)$ & 0.770 \\
\hline First care unit (\%) & & & 0.235 \\
\hline $\mathrm{ClCU}^{\mathrm{a}}$ & $40(15.7)$ & 44 (17.3) & \\
\hline SICUb & $174(68.5)$ & $154(60.6)$ & \\
\hline $\mathrm{MICU}$ & $33(13.0)$ & $48(18.9)$ & \\
\hline $\mathrm{NICU}$ & $7(2.8)$ & $8(3.1)$ & \\
\hline \multicolumn{4}{|l|}{ Ventilator (\%) } \\
\hline no & $101(39.8)$ & $42(16.5)$ & $<0.001$ \\
\hline yes & $153(60.2)$ & $212(83.5)$ & \\
\hline \multicolumn{4}{|l|}{ Vasopressor (\%) } \\
\hline no & $140(55.1)$ & $124(48.8)$ & 0.183 \\
\hline yes & $114(44.9)$ & $130(51.2)$ & \\
\hline CRRT (\%) & & & 0.247 \\
\hline no & 214 (84.3) & 203 (79.9) & \\
\hline yes & $40(15.7)$ & $51(20.1)$ & \\
\hline First PaO2/FiO2 & 134.77(83.00,219.76) & $113.06(75.00,170.00)$ & 0.004 \\
\hline Apache IV score & $75.50(60.25,96.75)$ & $78.50(58.00,99.75)$ & 0.680 \\
\hline SOFA score & $8.00(6.00,11.00)$ & $7.00(6.00,10.00)$ & 0.155 \\
\hline
\end{tabular}

ainclude Cardiac ICU, CCU-CTICU, CTICU, CSICU; binclude Med-sug ICU, SICU; ${ }^{c}$ represent Neuro ICU.

\section{Joint Model}

The distribution of longitudinal observation of 7,789 $\mathrm{PaO} 2 / \mathrm{FiO} 2$ measurements can be shown using trajectory function and plotted using interaction figures. Figure 3 shows the density of $\mathrm{PaO} 2 / \mathrm{FiO} 2$ values and the trajectory of each patient in different time periods. This plot indicates that most observations were concentrated in the first 5 days after patient admission. The result of multivariable Cox regression demonstrated that GCs treatment was associated with ICU mortality in ARDS patients [HR (95\% CI) $=0.642(0.453$, 0.912)], demonstrating that receiving GCs treatment is a protective factor in ARDS patients. The Survival curve also showed that GCs received group had lower risk of ICU mortality $(P=0.012$ ) (Figure 4). Linear mixed-effects models demonstrated that GCs treatment was not correlated to $\mathrm{PaO} 2 / \mathrm{FiO} 2$ trajectory after controlled by potential confounders. The result revealed that GCs had no effect on trend of $\mathrm{PaO} 2 / \mathrm{FiO} 2$ level. The JM combined the linear mixed-effects model and the Cox regression model to a less biased and fixed result for both models. The results indicated that after the adjustment, GCs treatment was still a protective factor for
ARDS patients $(\mathrm{HR}=0.602)$, indicating that patients who received GCs treatments had 39.8\% lower risk of mortality. In the JM, GCs was still not related to $\mathrm{PaO} 2 / \mathrm{FiO} 2$. The JM also demonstrated an association between $\mathrm{PaO} 2 / \mathrm{FiO} 2$ and the event status $(\mathrm{HR}=0.991)$, indicating that one unit increase in $\mathrm{PaO} 2 / \mathrm{FiO} 2$ will lower $0.9 \%$ of the ICU mortality risk of ARDS patients (Table 2).

\section{Marginal Structural Cox Model}

In our study, the MSCM included patients' overall $\mathrm{PaO} 2 / \mathrm{FiO} 2$, GCs treatment during ICU hospitalization, and baseline characteristics within $24 \mathrm{~h}$ after admission. SMDs after IPTW were $<0.2$ in both groups of virtual population. Baseline characteristics were shown in Supplementary Table 1. MSCM results showed that GCs use was associated with a significant improvement in ICU mortality in the ARDS population $[\mathrm{HR}(95 \% \mathrm{CI})=0.597(0.552,0.646) ; P<0.001]$. See Table 2.

\section{DISCUSSION}

In the present study, although the GCs was not significantly associated with the improvement of $\mathrm{PaO} 2 / \mathrm{FiO} 2$, under the Cox sub-model, the JM, and the MSCM results showed that receiving GCs may reduce the ICU mortality of patients with ARDS. And the longitudinal sub-model showed that increased $\mathrm{PaO} 2 / \mathrm{FiO} 2$ also had beneficial effects on the survival of ARDS patients. There is extensive evidence that the use of GCs can reduce systemic inflammation and accelerate the regression of ARDS, and it is also involved in adaptive lung repair and the improvement of extrapulmonary physiology $(21,22)$. The mechanism is that GCs can stimulate and promote the apoptosis of helper $\mathrm{T}$ cells, inhibit the production of pro-inflammatory cytokines, reduce the snowball effect of inflammatory response from the source (23), inhibit adhesion expression molecules, prevent them from rolling and adhesion in the inflammatory site, and weaken the chemotaxis of neutrophils (24). CCs can also induce membrane coupling protein expression and promote the separation and apoptosis of neutrophils, therefore inhibiting the inflammatory response and induce macrophage gene expression (25), increase macrophage phagocytic activity, improve natural immune function, and inhibit the excessive proliferation of capillaries and fibroblasts and the onset of pulmonary fibrosis (26). There are many studies supporting the effectiveness of GCs in the treatment of ARDS, for example, a multi-center study showed that early administration of GCs can reduce the duration of mechanical ventilation and overall mortality in patients with confirmed moderate to severe ARDS (27). A meta-analysis of 1703 COVID-19 patients showed systemic corticosteroid administration was associated with lower 28-day all-cause mortality (28). So GCs seem to be a treatment for acute respiratory distress syndrome. The oxygenation index is a vital sign for the diagnosis of ARDS. The Berlin definition is classified by hypoxia severity, suggesting that more-severe hypoxia increases the probability of mortality and the survival time with mechanical ventilation (29). JM result indicated similar 


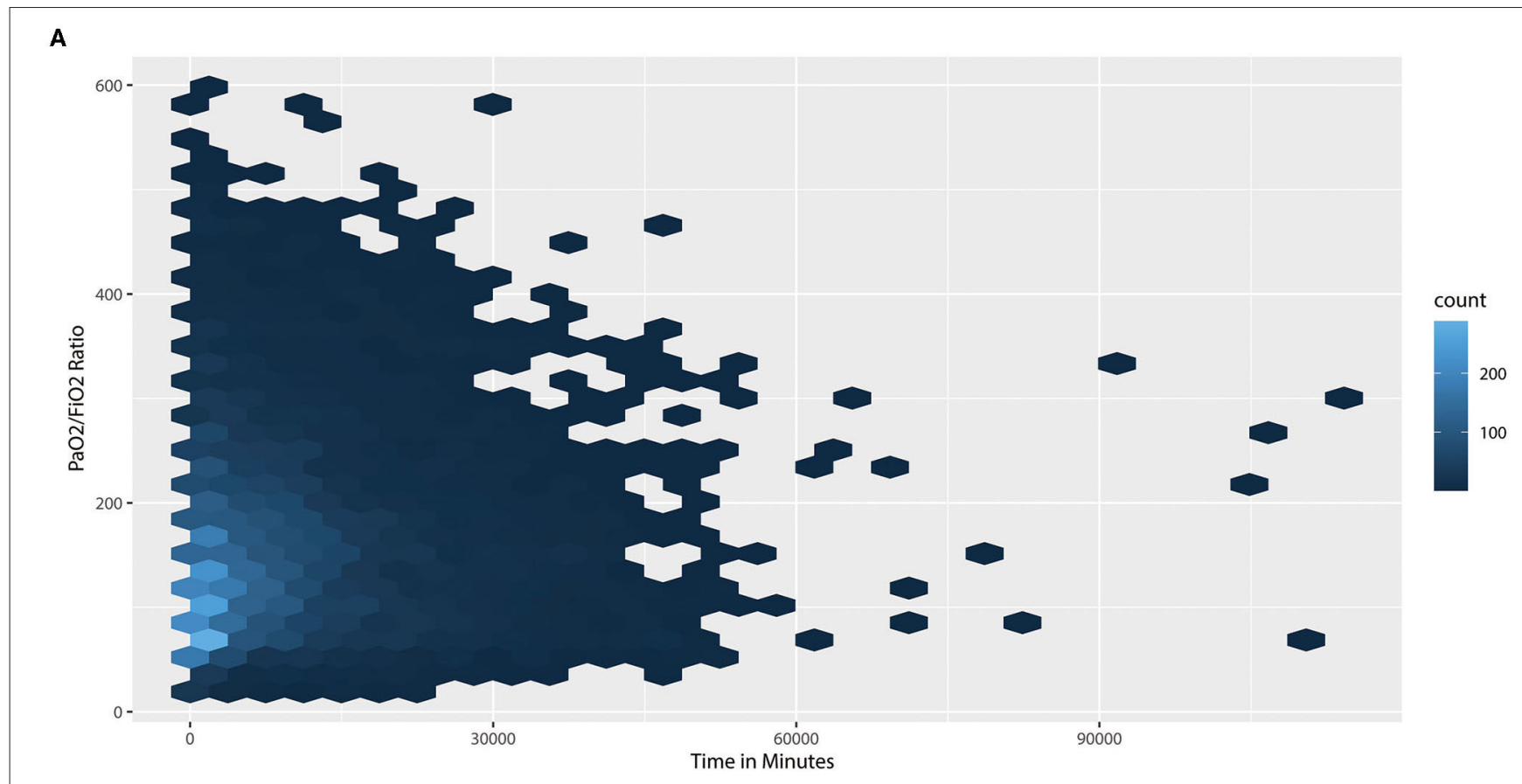

B

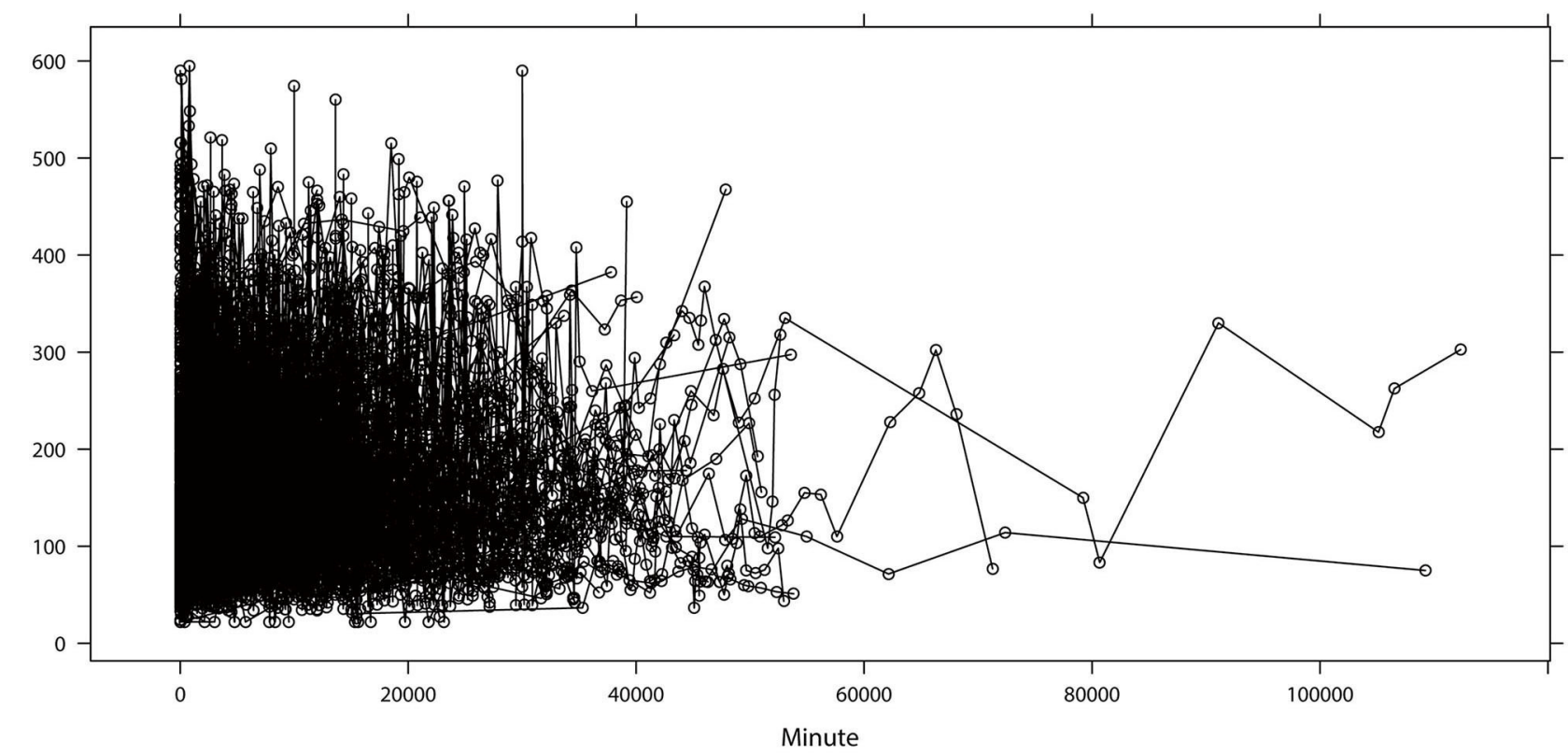

FIGURE 3 | The distribution of PaO2/FiO2 observations (A) and the trajectory (B) of PaO2/FiO2 Observations by Patients in Time Line. (A) showed the density of $\mathrm{PaO} 2 / \mathrm{FiO} 2$ ratio observations in time lines. (B) showed then trajectory of $\mathrm{PaO} 2 / \mathrm{FiO} 2$ for 508 patients, each line represented the one patient's trajectory.

trends, with the risk of death decreasing by $0.9 \%$ for each unit increase in $\mathrm{PaO} 2 / \mathrm{FiO} 2$. In our study, the use of GCs had no effect on $\mathrm{PaO} 2 / \mathrm{FiO} 2$ trends but the reduced $39.8 \%$ of mortality rate. Plenty of factors affect the oxygenation index, the most common factors include the concentration of inhaled oxygen and PEEP (30). In patients with ARDS, the primary goal of GCs therapy is to improve pulmonary and systemic inflammatory conditions, and the improvement of $\mathrm{PaO} 2 / \mathrm{FiO} 2$ may also require ventilator and other comprehensive treatments. Therefore, combining the GCs treatment and rational management of $\mathrm{PaO} 2 / \mathrm{FiO} 2$ could significantly increase the survival of ARDS patients. 


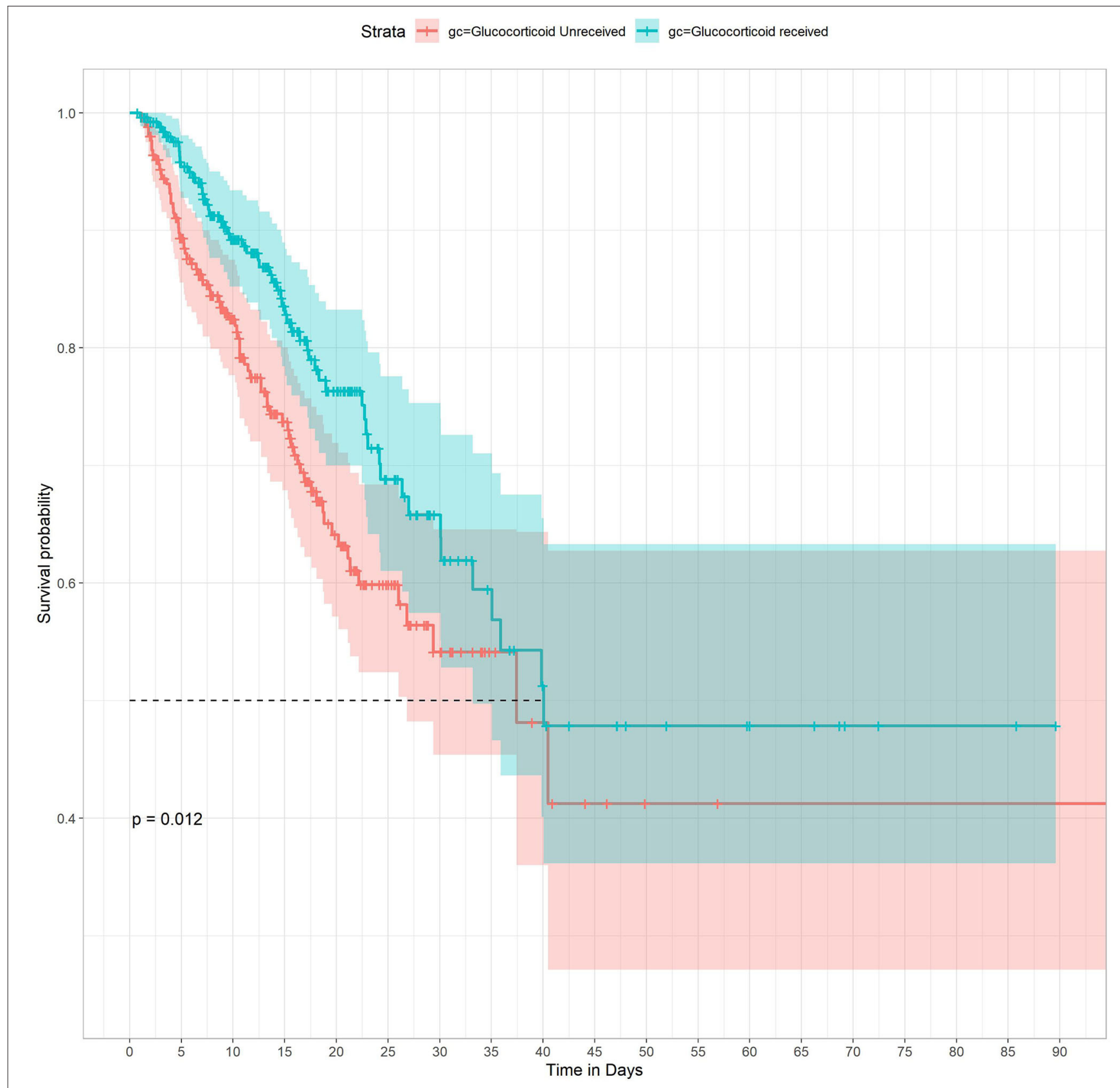

FIGURE 4 | Kaplan-Meier survival curves between groups. $P$-value calculated by Log-rank test $=0.012$ showed GCs received group had higher survival probability.

\section{STRENGTHS AND LIMITATIONS OF THE STUDY}

As a multi-center study, the results of this study are more representative and reliable. The matching results of PSM show that confounding factors are well-adjusted. Compared with independent Cox regression, JM has less bias and more accurate correlation coefficients, so the results of this article have higher reliability. In addition, we use the MSCM to further verify the results. Of course, this study only also had limitations. This research only analyzed whether patients received GCs treatment but did not specifically analyze the type, dose, and duration of GCs, which needs further analysis in the future.

\section{CONCLUSIONS}

The rational use of GCs therapy can reduce the ICU mortality of ARDS. Although GCs cannot improve $\mathrm{PaO} 2 / \mathrm{FiO} 2$, combining 
TABLE 2 | Result of effects between two sub-models, joint model and marginal structural cox model.

\begin{tabular}{|c|c|c|c|c|c|c|c|c|}
\hline & \multicolumn{2}{|l|}{$\operatorname{cox}$} & \multicolumn{2}{|l|}{ LME } & \multicolumn{2}{|l|}{ JM } & \multicolumn{2}{|l|}{ MSCM } \\
\hline Treatment effect on $\mathrm{PaO} 2 / \mathrm{FiO} 2$ & & & $0.001(0.000-11.491)$ & 0.150 & $0.000(0.000-1.359)$ & 0.060 & & \\
\hline $\mathrm{PaO} 2 / \mathrm{FiO} 2$ effect on survival & & & & & $0.991(0.987-0.995)$ & $<0.001$ & & \\
\hline
\end{tabular}

Cox, cox proportional hazards model; LME, linear mixed effect model; JM, joint model; MSCM: marginal structural cox model.

Coef, coefficient; SE, standard error. The hazard ratios (HRS) and 95\% confidence intervals (error bars) were calculated from Coef and SE.

Cox sub-model included: First admitted PaO2/FiO2 level and ventilator use.

LME sub-model included: GCs ${ }^{*}$ time, first admitted PaO2/FiO2 level, ventilator use.

MSCM included: Age, Sex, Race, BMI, First care unit, Ventilator use, Vasopressor use, CRRT, SOFA score, Apache IV score, overall PaO2/FiO2.

GCs treatment and increasing $\mathrm{PaO} 2 / \mathrm{FiO} 2$ can better improve patient survival.

\section{DATA AVAILABILITY STATEMENT}

The datasets presented in this study can be found in online repositories. The data were available on the eICU Collaborative Research Database at https://eicu-crd.mit.edu/.

\section{ETHICS STATEMENT}

The study was an analysis of a third-party anonymized publicly available database with pre-existing institutional review board (IRB) approval.

\section{AUTHOR CONTRIBUTIONS}

LZ created the study protocol, performed the statistical analyses, and wrote the first manuscript draft. ZW conceived the study and critically revised the manuscript. FX assisted with the study design and performed data collection.

\section{REFERENCES}

1. Yadav H, Thompson BT, Gajic O. Fifty Years of research in ARDS. Is acute respiratory distress syndrome a preventable disease? Am J Respir Crit Care Med. (2017) 195:725-36. doi: 10.1164/rccm.201609-1767CI

2. Thompson BT, Chambers RC, Liu KD. Acute respiratory distress syndrome. N Engl J Med. (2017) 377:562-72. doi: 10.1056/NEJMra1608077

3. Nieman GF, Andrews P, Satalin J, Wilcox K, Kollisch-Singule M, Madden M, et al. Acute lung injury: how to stabilize a broken lung. Crit Care. (2018) 22:136. doi: 10.1186/s13054-018-2051-8

4. Fan E, Brodie D, Slutsky AS. Acute respiratory distress syndrome: advances in diagnosis and treatment. JAMA. (2018) 319:698710. doi: 10.1001/jama.2017.21907

5. Dembinski R, Mielck F. [ARDS-an update-part 1: epidemiology, pathophysiology and diagnosis]. Anasthesiol Intensivmed Notfallmed Schmerzther. (2018) 53:102-11. doi: 10.1055/s-0043-107166

6. Butt Y, Kurdowska A, Allen TC. Acute lung injury: a clinical and molecular review. Arch Pathol Lab Med. (2016) 140:34550. doi: 10.5858/arpa.2015-0519-RA

7. Meduri GU, Marik PE, Chrousos GP, Pastores SM, Arlt W, Beishuizen A, et al. Steroid treatment in ARDS: a critical appraisal of the ARDS
YR assisted with data collection and manuscript editing. HW assisted the analysis and explain of statistical methods. DH confirmed the data and assisted with the statistical analyses. JL assisted with manuscript revision and data confirmation. HY contributed to data interpretation and manuscript revision. All authors read and approved the final manuscript.

\section{FUNDING}

This work was supported by the National Natural Science Foundation of China (No. 82072232; 81871585), the Natural Science Foundation of Guangdong Province (No. 2018A030313058), and Technology and Innovation Commission of Guangzhou Science, China (No.201804010308).

\section{SUPPLEMENTARY MATERIAL}

The Supplementary Material for this article can be found online at: https://www.frontiersin.org/articles/10.3389/fmed. 2021.678260/full\#supplementary-material network trial and the recent literature. Intensive Care Med. (2008) 34:619. doi: 10.1007/s00134-007-0933-3

8. Vandewalle J, Luypaert A, De Bosscher K, Libert C. Therapeutic Mechanisms of Glucocorticoids. Trends Endocrinol Metab. (2018) 29:42-54. doi: 10.1016/j.tem.2017.10.010

9. Villar J, Confalonieri M, Pastores SM, Meduri GU. Rationale for prolonged corticosteroid treatment in the acute respiratory distress syndrome caused by coronavirus disease 2019. Crit Care Explor. (2020) 2:e0111. doi: 10.1097/CCE.0000000000000111

10. Maláska J, Stašek J, Duška F, Balík M, Máca J, Hruda J, et al. Effect of dexamethasone in patients with ARDS and COVID-19-prospective, multicentre, open-label, parallel-group, randomised controlled trial (REMED trial): A structured summary of a study protocol for a randomised controlled trial. Trials. (2021) 22:172. doi: 10.1186/s13063-021-05116-9

11. Tomazini BM, Maia IS, Bueno FR, Oliveira Silva MVA, Baldassare FP, Vieira Costa EL, et al. COVID-19-associated ARDS treated with DEXamethasone (CoDEX): study design and rationale for a randomized trial. Rev Bras Terapia Intensiva. (2020) 32:354-62. doi: 10.5935/0103-507X.20200063

12. Tomazini BM, Maia IS, Cavalcanti AB, Berwanger O, Rosa RG, Veiga $\mathrm{VC}$, et al. Effect of dexamethasone on days alive and ventilator-free in patients with moderate or severe acute respiratory distress syndrome and 
COVID-19: the CoDEX randomized clinical trial. JAMA. (2020) 324:130716. doi: 10.1001/jama.2020.17021

13. Horby P, Lim WS, Emberson JR, Mafham M, Bell JL, Linsell L, et al. Dexamethasone in Hospitalized Patients with Covid-19. N Engl J Med. (2021) 384:693-704. doi: 10.1056/NEJMoa2021436

14. Zhang Z, Chen L, Ni H. The effectiveness of Corticosteroids on mortality in patients with acute respiratory distress syndrome or acute lung injury: a secondary analysis. Sci Rep. (2015) 5:17654. doi: 10.1038/srep17654

15. Li Y, Meng Q, Rao X, Wang B, Zhang X, Dong F, et al. Corticosteroid therapy in critically ill patients with COVID-19: a multicenter, retrospective study. Crit Care. (2020) 24:698. doi: 10.1186/s13054-020-03429-w

16. Ibrahim JG, Chu H, Chen LM. Basic concepts and methods for joint models of longitudinal and survival data. J Clin Oncol. (2010) 28:2796801. doi: 10.1200/JCO.2009.25.0654

17. Pollard TJ, Johnson AEW, Raffa JD, Celi LA, Mark RG, Badawi O. The eICU Collaborative research database, a freely available multi-center database for critical care research. Sci Data. (2018) 5:180178. doi: 10.1038/sdata.2018.178

18. Yang J, Li Y, Liu Q, Li L, Feng A, Wang T, et al. Brief introduction of medical database and data mining technology in big data era. J Evid Based Med. (2020) 13:57-69. doi: 10.1111/jebm. 12373

19. McCaffrey DF, Griffin BA, Almirall D, Slaughter ME, Ramchand R, Burgette LF. A tutorial on propensity score estimation for multiple treatments using generalized boosted models. Stat Med. (2013) 32:3388414. doi: $10.1002 / \operatorname{sim} .5753$

20. Feng M, McSparron JI, Kien DT, Stone DJ, Roberts DH, Schwartzstein RM, et al. Transthoracic echocardiography and mortality in sepsis: analysis of the MIMIC-III database. Intensive Care Med. (2018) 44:88492. doi: 10.1007/s00134-018-5208-7

21. Meduri GU, Bridges L, Shih MC, Marik PE, Siemieniuk RAC, Kocak M. Prolonged glucocorticoid treatment is associated with improved ARDS outcomes: analysis of individual patients' data from four randomized trials and trial-level meta-analysis of the updated literature. Intensive Care Med. (2016) 42:829-40. doi: 10.1007/s00134-0154095-4

22. Meduri GU, Annane D, Confalonieri M, Chrousos GP, Rochwerg B, Busby A, et al. Pharmacological principles guiding prolonged glucocorticoid treatment in ARDS. Intensive Care Med. (2020) 46:2284-96. doi: 10.1007/s00134-020-06289-8

23. Tu GW, Shi Y, Zheng YJ, Ju M-J, He H-Y, Ma G-G, et al. Glucocorticoid attenuates acute lung injury through induction of type 2 macrophage. J Transl Med. (2017) 15:181. doi: 10.1186/s12967-017-1284-7
24. Busillo JM, Cidlowski JA. The five Rs of glucocorticoid action during inflammation: ready, reinforce, repress, resolve, and restore. Trends Endocrinol Metab. (2013) 24:109-19. doi: 10.1016/j.tem.2012.11.005

25. Meduri GU, Annane D, Chrousos GP, Marik PE, Sinclair SE. Activation and regulation of systemic inflammation in ARDS: rationale for prolonged glucocorticoid therapy. Chest. (2009) 136:1631-43. doi: 10.1378/chest.08-2408

26. Rhen T, Cidlowski JA. Antiinflammatory action of glucocorticoidsnew mechanisms for old drugs. NEngl J Med. (2005) 353:171123. doi: 10.1056/NEJMra050541

27. Villar J, Ferrando C, Martínez D, Ambrós A, Muñoz T, Soler JA, et al. Dexamethasone treatment for the acute respiratory distress syndrome: a multicentre, randomised controlled trial. Lancet Respir Med. (2020) 8:267-76.

28. Sterne JAC, Murthy S, Diaz JV, Slutsky AS, Villar J, Angus DC, et al. Association Between Administration of Systemic Corticosteroids and Mortality Among Critically Ill Patients With COVID-19: A Meta-analysis. JAMA. (2020) 324:1330-41.

29. Ranieri VM, Rubenfeld GD, Thompson BT, Ferguson ND, Caldwell E, Fan E, et al. Acute respiratory distress syndrome: the Berlin Definition. JAMA. (2012) 307:2526-33. doi: 10.1001/jama.2012.5669

30. Narendra DK, Hess DR, Sessler CN, Belete HM, Guntupalli KK, Khusid F, et al. Update in management of severe hypoxemic respiratory failure. Chest. (2017) 152:867-79. doi: 10.1016/j.chest.2017.06.039

Conflict of Interest: The authors declare that the research was conducted in the absence of any commercial or financial relationships that could be construed as a potential conflict of interest.

Publisher's Note: All claims expressed in this article are solely those of the authors and do not necessarily represent those of their affiliated organizations, or those of the publisher, the editors and the reviewers. Any product that may be evaluated in this article, or claim that may be made by its manufacturer, is not guaranteed or endorsed by the publisher.

Copyright $\odot 2021$ Zhang, Wang, Xu, Ren, Wang, Han, Lyu and Yin. This is an open-access article distributed under the terms of the Creative Commons Attribution License (CC BY). The use, distribution or reproduction in other forums is permitted, provided the original author(s) and the copyright owner(s) are credited and that the original publication in this journal is cited, in accordance with accepted academic practice. No use, distribution or reproduction is permitted which does not comply with these terms. 